\title{
Sinergismo entre óleos essenciais e drogas antimicrobianas sobre linhagens de Staphylococcus aureus e Escherichia coli isoladas de casos clínicos humanos
}

\author{
Juliana A. A. Zago, Priscila I. Ushimaru, Lidiane N. Barbosa, Ary Fernandes Junior" \\ ${ }^{1}$ Departamento de Microbiologia e Imunologia, Instituto de Biociencias de Botucatu, Universidade Estadual \\ Paulista, Distrito Rubião Junior, 18.610-000 Botucatu-SP, Brasil
}

\begin{abstract}
RESUMO: Estudos com plantas e utilização em terapias combinatórias têm sido estimulados. Verificou-se as possíveis interações entre óleos essenciais de plantas [canela (Cinnamomum zeylanicum Blume Lauraceae), capim-cidreira (Cymbopogon citratus (DC.) Stapf, Poaceae), hortelãpimenta (Mentha piperita L. Lamiaceae), gengibre (Zingiber officinale Roscoe Zingiberaceae), cravo-da-índia (Caryophillus aromaticus L. Myrtaceae) e alecrim (Rosmarinus officinalis L. Lamiaceae)] combinados a oito drogas antimicrobianas frente a doze linhagens de Staphylococcus aureus e doze de Escherichia coli isoladas de humanos. Após determinação da Concentração Inibitória Mínima (CIM) para os óleos pelo método da diluição foram realizados ensaios para verificação de sinergismo entre os óleos essenciais e os antimicrobianos pela metodologia de Kirby \& Bauer. $S$. aureus foi mais suscetível às interações óleos e drogas, tendo o óleo de capim cidreira apresentado sinergismo com as oito drogas testadas, seguido pelo óleo de hortelã com sete drogas. Nos ensaios com E. coli, houve sinergismo apenas para os óleos de alecrim (três drogas) e capimcidreira (duas drogas). Não ocorreram casos de antagonismo e os resultados de sinergismo foram influenciados pelos microrganismos estudados.
\end{abstract}

Unitermos: Drogas antimicrobianas, bactérias, óleos essenciais, plantas medicinais, sinergismo.

\begin{abstract}
Synergism between essential oils and antimicrobial drugs against Staphylooccus aureus and Escherichia coli strains from human infections". The studies with plants and combinatory therapy have been stimulated. The interactions between cinnamon (Cinnamomun zeylanicum Blume Lauraceae), lemon grass (Cymbopogon citratus (DC.) Stapf, Poaceae), mint (Mentha piperita L. Lamiaceae), ginger (Zingiber officinale Roscoe Zingiberaceae), clove (Caryophillus aromaticus L. Myrtaceae) and rosemary (Rosmarinus officinalis L. Lamiaceae) and eight antimicrobial drug was carried. It was made against twelve S. aureus and twelve E.coli strains isolated from human specimens. After minimal inhibitory concentration (MIC) values determination of essential oils by dilution agar method, the synergism assays were performed by Kirby and Bauer method. The $S$. aureus was susceptible to oils and drugs interactions, and the lemon grass oils showed synergism with all drugs tested followed by mint with seven drugs. E.coli assays, synergism was observed only with rosemary (three drugs) and lemon grass (two drugs). No antagonism between drugs and oils tested was observed and the results were variable according to microorganism used.
\end{abstract}

Keywords: Antimicrobial drugs, bacteria, essential oils, medicinal plants, synergism.

\section{INTRODUÇÃO}

O uso de plantas medicinais é uma prática antiga e atualmente é predominante em países em desenvolvimento, onde existe uma dependência na denominada medicina popular como solução alternativa para problemas de saúde, especialmente na Ásia, África e América Latina (Sartoratto et al., 2004). A tradição popular também serve como base a farmacologia moderna, sendo que parte das drogas sintéticas atuais tem origem, direta ou indireta, nas plantas medicinais (Silver \& Bostian, 1993).

A propriedade antimicrobiana é motivo de inúmeros estudos devido ao aumento da resistência bacteriana às drogas antimicrobianas convencionais, além do que também são necessários estudos visando entendimento desta propriedade, mesmo para as plantas que já tiveram seu potencial antimicrobiano comprovado (Schelz \& Hohmann, 2006). Também já foi verificado que o uso associado de plantas medicinais, além dos seus compostos derivados, e drogas antimicrobianas pode inibir ou intensificar o efeito terapêutico das drogas antimicrobianas convencionais, bem como não interferir na resposta esperada (Nascimento et al., 2000).

Os óleos essenciais são responsáveis por conferir 
aroma e sabor característico às plantas e são relacionados à atração de polinizadores, proteção contra insetos e diversas funções necessárias à sobrevivência da planta (Lima et al., 2003; Santos et al., 2004), eles apresentam composição complexa, sendo os terpenos considerados a classe de substâncias mais encontrada nas plantas (Santos et al., 2004). A composição química do óleo pode apresentar variação devida à fatores ambientais e de manejo das plantas bem como da forma de extração e armazenamento, interferindo na atividade antimicrobiana (Nascimento et al., 2007).

A escolha da planta para utilização do seu óleo essencial é feita tendo como critério o seu uso terapêutico e etnobotânico, presença de determinadas substâncias ativas ou de acordo com sua disponibilidade (Maciel et al., 2002). Óleo essencial de alecrim apresenta terpenos, especialmente $\alpha$-pinemo e canfemo (Angioni et al., 2004), sendo a ação antimicrobiana verificada frente a linhagens bacterianas e também sobre linhagens de leveduras (Schelz \& Hohmann, 2006). Mentol, mentona, mentofurano, terpineno, limoneno, cineol, felandreno e $\alpha$-pinemo são as principais substâncias do óleo essencial da hortelã pimenta. O óleo essencial de capim-cidreira (Cymbopogum citratus (DC.) Stapf) é composto de mirceno, neral, geranial e outras substâncias e é utilizado na medicina popular para o tratamento de resfriados, disenteria, dores de cabeça, tranqüilizante e antiespasmódico (Pereira et al., 2004), sendo frequentes os relatos sobre sua ação antimicrobiana (Nguefack et al., 2004). O uso do gengibre (Zingiber officinale Roscoe) é bem conhecido na culinária como tempero e há muito tempo os chineses já o utilizavam na medicina. Geralmente utilizado no tratamento de disenteria, malária, reumatismo e resfriados, seu óleo é composto por uma diversidade de terpenos (Sabulal et al., 2006). O óleo essencial de canela (Cinnamomum zeylanicum Blume) é utilizado como flavorizante, aromatizante e conservante natural de alimentos e estudos mostraram a capacidade de inibir fungos (Lima et al., 2006) e bactérias (Matan et al., 2006). Foramidentificados 23 constituintes noóleo essencial obtido de folhas de canela, sendo o eugenol a substância que apresentou maior percentual (60\%). A partir de galhos foram identificados 36 substâncias com predominância de monoterpenos $\alpha$ e $\beta$-pineno, $\alpha$-felandreno, $p$-cimeno, limoneno, linalol, sequiterpenos $\alpha$-copaeno, $\beta$-cariofileno, oxido de cariofileno e os alilbenzenos $\varepsilon$-cinamaldeido e aceto de $\varepsilon$-cinamila (Lima et al., 2005). O cravo-daíndia (Caryophillus aromaticus), pertencente à família Myrtaceae, e seu óleo essencial está presente na planta em grande quantidade, entre 15 e $25 \%$, tendo como principal constituinte o eugenol (Silva, 2006), sendo esta substância de reconhecida atividade antibacteriana e antifúngica, e também o mais ativo quando testado frente a linhagens de E. coli (Burt \& Reinders, 2003; Rhayour et al., 2003).

Assim, este trabalho buscou estudar as possíveis interações entre determinados óleos essenciais extraídos de plantas consideradas medicinais, como a canela
(Cinnamomun zeylanicum), o capim-cidreira (Cymbopogon citratus), a hortelã (Mentha piperita), o gengibre (Zingiber officinalle), o cravo-da-índia (Caryophillus aromaticus) e o alecrim (Rosmarinus Officinalis), com determinadas drogas antimicrobianas; frente às linhagens de Staphylococcus aureus e Escherichia coli isoladas de casos clínicos humanos através de adaptação da metodologia de Kirby \& Bauer.

\section{MATERIAL E MÉTODOS}

\section{Plantas medicinais e óleos essenciais}

As amostras de cravo-da-índia e gengibre foram obtidas no comercio local da cidade de Botucatu-SP e a amostra de capim-cidreira no canteiro de plantas medicinais localizado na Faculdade de Ciências Agronômicas, UNESP, Botucatu, sendo a exsicata depositada no Herbário do Departamento de Botânica do Instituto de Biociências, UNESP, Botucatu sob numero BOTU 25663. Os óleos foram obtidos pela metodologia do arraste com vapor de água no laboratório do Departamento de Microbiologia e Imunologia do Instituto de Biociências, UNESP, campus de Botucatu. Os óleos das demais plantas foram obtidos em empresa especializada na comercialização de produtos desta natureza (Bioessência Produtos Naturais Ltda, Barra Bonita-SP).

\section{Interações entre óleos essenciais e drogas antimicrobianas}

Foram testadas linhagens bacterianas de Staphylococcus aureus $(\mathrm{n}=12)$ e Escherichia coli $(\mathrm{n}=$ 12) isoladas a partir de materiais biológicos de pacientes do Hospital das Clínicas da Faculdade de Medicina da Universidade Estadual Paulista, UNESP, Campus de Botucatu. Previamente as linhagens foram semeadas em meio de Agar Sangue e MacConkey para verificação de viabilidade e pureza das linhagens de $S$. aureus e E. coli, respectivamente.

Os valores de Concentração Inibitória Mínima (CIM) utilizados na realização dos ensaios sobre interações de drogas foram obtidos previamente utilizando a metodologia da diluição dos óleos essenciais em meio de Müeller Hinton Agar (MHA) e posterior cálculo da CIM 90\% (NCCLS, 2003; Betoni et al., 2006). Estes valores são apresentados na Tabela 1 juntamente com os valores de $1 / 4$ CIM 90, que foram utilizados nos ensaios para verificação de possíveis interações entre os óleos essenciais e as drogas antibacterianas. A metodologia adotada foi a dos discos, preconizadas por Kirby \& Bauer (CLSI, 2005) e adaptada por Betoni et al. (2006).

Foram realizados dois tipos de antibiogramas: um controle e um tratamento. Nos tratamentos, os óleos essenciais das plantas foram diluídos $(\% \mathrm{v} / \mathrm{v})$ em meio de Mueller Hinton Ágar (MHA) obtendo-se concentrações 
correspondentes à proporção de $1 / 4$ da CIM 90\%, de acordo com valores obtidos previamente para cada um dos óleos essenciais em estudo (Tabela 1). Para possibilitar a diluição dos óleos essenciais no meio de cultura, foi utilizado Tween 80 na proporção de $0,2 \%$ v/v (Nascimento et al., 2007). Após incubação $\left(37^{\circ} \mathrm{C} / 24 \mathrm{~h}\right)$, os halos de inibição foram anotados (milímetros) e na análise estatística foram comparados os valores obtidos nos ensaios controles e tratamentos. Os experimentos foram realizados em duplicatas e utilizados para análise estatística a média dos valores observados. As drogas antimicrobianas testadas foram: cloranfenicol $(30 \mu \mathrm{g})$, gentamicina $(10 \mu \mathrm{g})$, cefepima $(10 \mu \mathrm{g})$, tetraciclina $(30 \mu \mathrm{g})$, sulfazotrim $(25 \mu \mathrm{g})$, cefalotina $(30 \mu \mathrm{g})$, ciprofloxacina $(5 \mu \mathrm{g})$ e rifampicina $(5$ $\mu \mathrm{g})$.

\section{Análise estatística}

Para os resultados obtidos através do método de Kirby \& Bauer para cada bactéria, foi utilizado o teste de Mann-Whitney para a verificação de possíveis interações entre o óleo essencial (tratamento) e as drogas antibacterianas. Quando a diferença entre a mediana do controle e a do tratamento, para cada droga, era significativa ( $\mathrm{p} \leq 0,05)$, concluía-se a ocorrência de interação.

Tabela 1. Valores de CIM 90\% e 1/4 CIM 90 expressos em \% v/v para $S$. aureus e E. coli.

\begin{tabular}{ccccccc}
\hline Bactérias & Canela \% v/v & Alecrim \% v/v & Hortelã $\%$ v/v & Cravo \% v/v & Gengibre \% v/v & Capim cidreira \% v/v \\
\hline S. aureus & & & & & & \\
CIM90\% & 0,047 & 0,550 & 0,280 & 0,095 & 0,090 & 0,100 \\
$1 / 4$ CIM90\% & 0,011 & 0,140 & 0,070 & 0,024 & 0,022 & 0,025 \\
\hline E.coli & & & & & & 0,520 \\
CIM90\% & 0,090 & 5,000 & 1,240 & 0,083 & 0,130 & 0,140 \\
$1 / 4$ CIM90\% & 0,022 & 1,250 & 0,310 & 0,021 & 0 \\
\hline
\end{tabular}

\section{RESULTADOS E DISCUSSÃO}

Nas Tabelas 2 e 3 são apresentados os valores das medianas encontrados para cada tratamento de acordo com as drogas antimicrobianas, comparando-os com os valores obtidos no antibiograma controle. Através desses resultados, aplicando-se a análise estatística pelo teste de Mann-Whitney, pode-se observar a interferência dos óleos essenciais sobre o poder antibacteriano das drogas testadas (Tabela 4). As linhagens de S. aureus mostraram-se mais suscetíveis à interferência dos óleos sobre as drogas antimicrobianas testadas, tendo verificado 26 eventos (54\%) de interações do tipo sinergismo, para um total de 48 combinações possíveis (oito drogas x seis óleos). Em estudo realizado por Oliveira et al. (2006), as bactérias Gram-positivas ( $S$. aureus e $S$. epidermis) se mostraram mais sensíveis às interações entre drogas antibacterianas e óleos essenciais selecionados para o estudo. Uma explicação é que as bactérias Gram positivas são mais sensíveis à ação de agentes antimicrobianos devido à presença de uma parede bacteriana que normalmente não restringe a penetração de moléculas tóxicas, enquanto as Gram negativas possuem um sistema de barreira constituído pela membrana externa da parede bacteriana formada por fosfolipídios, lipopolissacarídeos e proteínas (porinas) que conferem considerável impermeabilidade aos agentes antibacterianos, resultando em maior resistência dessas bactérias aos antibióticos (Lambert, 2002).

Tabela 2. Valores das medianas encontrados pelo método de Kirby \& Bauer para o controle (C) e para os tratamentos (T) com óleos essenciais frente às drogas antibacterianas para 12 linhagens de $S$. aureus.

\begin{tabular}{ccccccccccccc}
\hline & \multicolumn{2}{c}{ Canela } & \multicolumn{2}{c}{ Alecrim } & \multicolumn{2}{c}{ Hortelã } & \multicolumn{2}{c}{ Cravo } & \multicolumn{2}{c}{ Gengibre } & Capim \\
\hline Drogas & C & T & C & T & C & T & C & T & C & T & C & T \\
\hline CLO & 23,0 & 24,0 & 23,0 & 24,0 & $\mathbf{2 3 , 0}$ & $\mathbf{2 6 , 5}$ & 23,0 & 25,0 & $\mathbf{2 3 , 0}$ & $\mathbf{2 4 , 5}$ & $\mathbf{2 3 , 0}$ & $\mathbf{2 6 , 0}$ \\
CFL & 30,0 & 31,0 & 30,0 & 30,5 & $\mathbf{3 0 , 0}$ & $\mathbf{3 3 , 0}$ & 30,0 & 32,0 & $\mathbf{3 0 , 0}$ & $\mathbf{3 3 , 0}$ & $\mathbf{3 0 , 0}$ & $\mathbf{3 9 , 0}$ \\
CPM & 24,5 & 25,5 & 24,5 & 25,0 & 24,5 & 25,5 & 24,5 & 24,5 & 24,5 & 26,0 & $\mathbf{2 4 , 5}$ & $\mathbf{3 0 , 0}$ \\
GEN & 23,0 & 25,0 & $\mathbf{2 3 , 0}$ & $\mathbf{2 5 , 5}$ & $\mathbf{2 3 , 0}$ & $\mathbf{2 5 , 5}$ & $\mathbf{2 3 , 0}$ & $\mathbf{2 7 , 0}$ & $\mathbf{2 3 , 0}$ & $\mathbf{2 6 , 0}$ & $\mathbf{2 3 , 0}$ & $\mathbf{3 1 , 0}$ \\
TET & 24,0 & 25,0 & $\mathbf{2 4 , 0}$ & $\mathbf{2 6 , 0}$ & $\mathbf{2 4 , 0}$ & $\mathbf{2 7 , 0}$ & 24,0 & 27,0 & $\mathbf{2 4 , 0}$ & $\mathbf{2 6 , 5}$ & $\mathbf{2 4 , 0}$ & $\mathbf{2 9 , 0}$ \\
SUT & 28,0 & 29,0 & $\mathbf{2 8 , 0}$ & $\mathbf{2 9 , 5}$ & $\mathbf{2 8 , 0}$ & $\mathbf{3 0 , 0}$ & 28,0 & 29,0 & 28,0 & 29,0 & $\mathbf{2 8 , 0}$ & $\mathbf{3 0 , 5}$ \\
RIF & 25,5 & 27,0 & 25,5 & 27,0 & $\mathbf{2 5 , 5}$ & $\mathbf{2 8 , 0}$ & $\mathbf{2 5 , 5}$ & $\mathbf{2 8 , 0}$ & 25,5 & 27,0 & $\mathbf{2 5 , 5}$ & $\mathbf{3 1 , 5}$ \\
CIP & 28,0 & 29,5 & 28,0 & 29,0 & $\mathbf{2 8 , 0}$ & $\mathbf{3 1 , 0}$ & $\mathbf{2 8 , 0}$ & $\mathbf{3 0 , 5}$ & $\mathbf{2 8 , 0}$ & $\mathbf{3 0 , 5}$ & $\mathbf{2 8 , 0}$ & $\mathbf{3 5 , 0}$ \\
\hline
\end{tabular}

CLO: cloranfenicol, CFL: cefalotina, CPM: cefepime, GEN: gentamicina, TET: tetraciclina, SUT: sulfazotrim, RIF: rifampicina, CIP: ciprofloxacina.Valores em negrito quando diferença significativa $(\mathrm{p} \leq 0,05)$ 
Tabela 3. Valores das medianas encontrados pelo método de Kirby\&Bauer para o controle (C) e para os tratamentos (T) com óleos essenciais frente às drogas antibacterianas para 12 linhagens de E. coli. .

\begin{tabular}{ccccccccccccc}
\hline & \multicolumn{2}{c}{ Canela } & \multicolumn{2}{c}{ Alecrim } & \multicolumn{2}{c}{ Hortelã } & \multicolumn{2}{c}{ Cravo } & \multicolumn{2}{c}{ Gengibre } & \multicolumn{2}{c}{ Capim } \\
\hline Drogas & C & T & C & T & C & T & C & T & C & T & C & T \\
\hline CLO & 21,0 & 24,0 & 21,0 & 28,5 & 21,0 & 22,5 & 21,0 & 23,0 & 21,0 & 25,0 & 21,0 & 25,0 \\
CFL & 16,0 & 16,0 & 16,0 & 15,5 & 16,0 & 16,5 & 16,0 & 15,5 & 16,0 & 16,5 & 16,0 & 19,0 \\
CPM & 31,5 & 30,5 & 31,5 & 33,5 & 31,5 & 32,0 & 31,5 & 30,0 & 31,5 & 33,0 & 31,5 & 35,0 \\
GEN & 21,5 & 21,5 & 21,5 & 22,0 & 21,5 & 21,5 & 21,5 & 21,5 & 21,5 & 21,0 & 21,5 & 21,5 \\
TET & 14,5 & 15,5 & 14,5 & 23,5 & 14,5 & 19,0 & 14,5 & 19,0 & 14,5 & 14,5 & 14,5 & 18,0 \\
SUT & 26,0 & 26,5 & 26,0 & 29,0 & 26,0 & 28,5 & 26,0 & 28,5 & 26,0 & 26,5 & 26,0 & 28,0 \\
RIF & 12,5 & 14,5 & 12,5 & 14,5 & 12,5 & 13,0 & 12,5 & 13,0 & 12,5 & 9,0 & 12,5 & 9,5 \\
CIP & 31,5 & 32,5 & 31,5 & 34,5 & 31,5 & 33,5 & 31,5 & 33,5 & 31,5 & 33,5 & 31,5 & 34,5 \\
\hline
\end{tabular}

CLO: Cloranfenicol, CFL: Cefalotina, CPM: Cefepime, GEN: Gentamicina, TET: Tetraciclina, SUT: Sulfazotrim, RIF: Rifampicina, CIP: Ciprofloxacina.Valores em negrito com diferença significativa $\mathrm{p} \leq 0,05$

Tabela 4. Taxa de sinergismo entre drogas antimicrobianas e óleos essenciais junto a 13 linhagens $S$. aureus e E.coli pelo método de Kirby \& Bauer.

\begin{tabular}{|c|c|c|c|c|c|c|c|c|c|c|c|c|c|c|}
\hline & \multicolumn{2}{|c|}{ Canela } & \multicolumn{2}{|c|}{ Alecrim } & \multicolumn{2}{|c|}{ Hortelã } & \multicolumn{2}{|c|}{ Cravo } & \multicolumn{2}{|c|}{ Gengibre } & \multicolumn{2}{|c|}{ Capim } & \multicolumn{2}{|c|}{ Sinergismo } \\
\hline & S. aureus & E. coli & S. aureus & E. coli & S. aureus & E. coli & S. aureus & E. coli & S. aureus & E. coli & S. aureus & E. coli & S. aureus & E. coli \\
\hline CLO & - & - & - & $X$ & $X$ & - & - & - & $\mathrm{X}$ & - & $\mathrm{X}$ & - & 3 & 1 \\
\hline CFL & - & - & - & - & $\mathrm{X}$ & - & - & - & $\mathrm{X}$ & - & $\mathrm{X}$ & $\mathrm{X}$ & 3 & 1 \\
\hline CPM & - & - & - & $\mathrm{X}$ & - & - & - & - & - & - & $\mathrm{X}$ & $\mathrm{X}$ & 1 & 2 \\
\hline GEN & - & - & $\mathrm{X}$ & - & $\mathrm{X}$ & - & $\mathrm{X}$ & - & $\mathrm{X}$ & - & $\mathrm{X}$ & - & 5 & 0 \\
\hline TET & - & - & $\mathrm{X}$ & $\mathrm{X}$ & $\mathrm{X}$ & - & - & - & $\mathrm{X}$ & - & $\mathrm{X}$ & - & 4 & 1 \\
\hline SUT & - & - & $\mathrm{X}$ & - & $\mathrm{X}$ & - & - & - & - & - & $\mathrm{X}$ & - & 3 & 0 \\
\hline RIF & - & - & - & - & $\mathrm{X}$ & - & $\mathrm{X}$ & - & - & - & $\mathrm{X}$ & - & 3 & 0 \\
\hline CIP & - & - & - & - & $\mathrm{X}$ & - & $\mathrm{X}$ & - & $\mathrm{X}$ & - & $\mathrm{X}$ & - & 4 & 0 \\
\hline Total & 0 & 0 & 3 & 3 & 7 & 0 & 3 & 0 & 5 & 0 & 8 & 2 & & \\
\hline
\end{tabular}

(X): Sinergismo quando $\mathrm{p} \leq 0,05$; (-): Indiferente. CLO: Cloranfenicol, CFL: Cefalotina, CPM: Cefepime, GEN: Gentamicina, TET: Tetraciclina, SUT: Sulfazotrim, RIF: Rifampicina, CIP: Ciprofloxacina.

O óleo essencial de capim cidreira apresentou sinergismo com as oito drogas testadas nos ensaios com S. aureus, seguido pela hortelã que apresentou sinergismo com sete drogas e pelo gengibre com cinco drogas. O óleo essencial de canela não apresentou qualquer tipo de interação. A gentamicina apresentou sinergismo com o maior número de óleos testados, sendo cinco dos seis testados enquanto a cefepime apresentou apenas um caso de sinergismo com o óleo de capim cidreira.

Nos ensaios para E. coli ocorreram apenas cinco interações com sinergismo entre os óleos essenciais e as drogas testadas, o que correspondeu a apenas $10,4 \%$ do total possível de interações, sendo os óleos de alecrim e capimcidreira os que interferiram na ação antimicrobiana das drogas selecionadas sendo três e dois casos de sinergismo respectivamente. Quanto às drogas antimicrobianas, e contrário ao que ocorreu nos ensaios com $S$. aureus, a cefepime apresentou o maior numero de sinergismo verificado com dois dos óleos testados. Também não foram observados casos de antagonismo e confirmou-se a tendência de maior resistência aos antimicrobianos das linhagens Gram-negativas (Denyer \& Maillard, 2002; Lambert, 2002).

Pelos resultados apresentados na Tabela 2, observa-se uma tendência de interação positiva entre determinadas drogas e óleos essenciais. Para a $S$. aureus, as drogas gentamicina, cefalotina e cefepime tiveram seu perfil de sensibilidade melhorado quando expostas aos tratamentos com óleo essencial de capim cidreira e de hortelã. Seguidos pelos óleos de cravo da Índia e gengibre que interferiram na gentamicina. Em relação a E. coli, o cloranfenicol apresentou um aumento considerável no número de linhagens sensíveis quando este foi testado junto com o óleo essencial de capim cidreira e hortelã. Esse aumento também foi verificado nos óleos de alecrim e hortelã para a tetraciclina.

Assim, o estudo indica possibilidade do uso de produtos naturais combinados aos antimicrobianos tradicionais, no intuito de aumentar o potencial antimicrobiano das drogas. Vale destacar também que mesmo os óleos que apresentaram um efeito antimicrobiano não tão eficiente como, por exemplo, os óleos essenciais de 
capim cidreira, alecrim e hortelã, quando foram avaliados nos ensaios para determinação de sinergismo, estes foram para S. aureus os que apresentaram maior índice de sinergismo como, por exemplo, no caso do capim-cidreira que mostrou sinergismo com $100 \%$ das drogas testadas. Este mesmo fenômeno foi relatado com extrato de capim cidreira, que mesmo apresentando ação antimicrobiana fraca, foi o que apresentou maior índice de sinergismo com drogas antimicrobianas nos ensaios in vitro (Betoni et al., 2006).

Outro aspecto importante do estudo foi que a espécie do microrganismo avaliado interfere com os resultados, verificado pela baixa frequência de sinergismo dos óleos e drogas nos ensaios com as linhagens de E. coli. Isto reforça o fato que mais estudos in vitro são necessários visando estabelecer o perfil de sinergismo frente outras espécies bacterianas, pois se assim não for feito é possível a ocorrência de falhas quando for utilizada terapia combinatória no tratamento de doenças infecciosas. Ríos \& Recio (2005) acrescentaram que os estudos de plantas medicinais como agentes antimicrobianos devem ser focados para a obtenção do maior número de informações específicas possíveis, como, por exemplo, os mecanismos de ações que possibilitam a sua ação antimicrobiana, bem como a toxicidade para células animais ou humanas, os efeitos in vivo, as possíveis interações positivas ou negativas com os antibióticos tradicionais, entre outras propriedades, além de padronização na metodologia visando possibilitar a reprodutibilidade nos ensaios biológicos. Também é fundamental que a composição do óleo essencial seja precisamente conhecida, pois, como relatado em diversos estudos, sua composição pode variar de acordo com diversos fatores, como o clima, solo, época e forma de plantio, adubação, condições ambientais, técnica de extração, além de variações genéticas intra-específicas da espécie vegetal, entre outros fatores que podem alterar o teor do princípio ativo presente no óleo (Nascimento et al., 2007).

Assim, é possível concluir que o $S$. aureus é mais suscetível às interações quando comparado com a $E$. coli e que o óleo essencial do capim-cidreira apresentou sinergismo com o maior número de drogas testadas. As drogas que mostraram maior índice de sinergismo com os vários óleos testados foram gentamicina, tetraciclina e ciprofloxacina, nos ensaios com $S$. aureus e cefepime nos ensaios com E. coli.

\section{REFERÊNCIAS}

Angioni A, Barra A, Cereti E, Barile D, Coisson JD, Arlorio M, Dessi S, Coroneo V, Cabras P 2004. Chemical composition, plant genetic differences, antimicrobial and antifungal activity investigation of the essential oil of Rosmarinus officinalis L. J Agr Food Chem 52: 35303535.

Betoni JEC, Mantovani RP, Barbosa LN, Di Stasi LC, Fernandes
Junior A 2006. Synergism between plants extract and antimicrobial drugs used on Staphylococcus aureus diseases. Mem Inst Oswaldo Cruz 101: 387-390.

Burt SA, Reinders RD 2003. Antibacterial activity of selected plant essential oils against Escherichia coli O157:H7. Lett Appl Microbiol 36: 162-167.

Clinical and Laboratory Standards Institute (CLSI) 2005. Performance standards for antimicrobial susceptibility testing. 5. informational supplement. CLSI document M100-S15. Wayne (PA).

Denyer SP, Maillard JY 2002. Cellular impermeability and uptake of biocides and antibiotics in Gram-negative bacteria. $J$ Appl Microbiol Symposium Supplement 92: 35S-45S.

Lambert PA 2002. Cellular impermeability and uptake of biocides and antibiotics in Gram-positive bactéria and mycobacteria. J Appl Microbiol Symposium Supplement 92: 46S-54S

Lima EO, Farias NMP, Souza EL, Santos BHC 2003. Propriedades antibacterianas de óleos essenciais de plantas medicinais. Rev Bras Cienc Saude 7: 251-258.

Lima IO, Oliveira RAG, Lima EO, Farias NMP, Souza EL 2006. Antifungal activity from essential oils on Candida species. Rev Bras Farmacogn 16: 197-201.

Lima MP, Zoghbi MGB, Andrade EHA, Silva TMD, Fernandes CS 2005. Constituintes voláteis das folhas e dos galhos de Cinnamonum zeylanicum Blume (Lauraceae). Acta Amaz 35: 363-366.

Maciel MAM, Pinto AC, Veiga Jr VF 2002. Medicinal plants: The need for multidisciplinary scientific studies. Quim Nova 25: 429-438.

Matan N, Rimkeeree H, Mawson AJ, Chompreeda P, Haruthaithanasan V, Parker M 2006. Antimicrobial activity of cinnamon and clove oils under modified atmosphere conditions. Int J Food Microbiol 107: 180185

Nascimento GGF, Lucatelli J, Freitas PC, Silva GL 2000. Antibacterial activity of plant extracts and phytochemicals on antibiotic-resistant bacteria. Braz J Microbiol 31: 247-256.

Nascimento PFC, Nascimento AC, Rodrigues CS, Antoniolli AA, Santos PO, Barbosa Junior AM, Trindade R.C 2007. Antimicrobial activity of the essentials oils: a multifactor approach of the methods. Rev Bras Farmacogn 17: 108113.

National Commitee for Clinical Laboratory Standards (NCCLS) 2003. Method for dilution antimicrobial susceptibility tests for bacteria that grow aerobically. 6. ed. Wayne (PA): Approved Standard M7-A6.

Nguefack J, Budde BB, Jakobsen M 2004. Five essential oils from aromatic plants of Cameroon: their antibacterial activity and ability to permeabilize the cytoplasmic membrane of Listeria innocua examined by flow cytometry. Lett Appl Microbiol 39: 395-400.

Pereira RS, Sumita TC, Furlan MR, Jorge AOC, Ueno M 2004. Antibacterial activity of essential oils on microorganisms isolated from urinary tract infection. Rev Saude Publica 
38: 326-328.

Oliveira RAG, Lima EO, Vieira WL, Freire KRL, Trajano VN, Lima IO, Souza EL, Toledo MS, Silva Filho RN 2006. Estudo da interferência de óleos essenciais sobre a atividade de alguns antibióticos usados na clínica. Rev Bras Farmacogn 16: 77-82.

Rhayour K, Bouchikhi T, Tantaoui-Elaraki A, Remmal A 2003. The mechanism of bactericidal action of oregano and clove essential oils and of their phenolic major components on Escherichia coli and Bacillus subtilis. $J$ Essent Oil Res 15: 356-362.

Ríos JL, Recio MC 2005. Medicinal plants and antimicrobial activity. J Ethnopharmacol 100: 80-84.

Santos AS, Alves SM, Figueiredo FJC, Rocha Neto OG 2004. Descrição de sistema e métodos de extração de óleos essenciais e determinação de umidade de biomassa em laboratório. Comunicado Técnico-Embrapa 99: 1-6.

Sabulal B, Dan M, J AJ, Kurup R, Pradeep NS, Valsamma RK, George V 2006. Caruophyllene-rich rhizome oil of Zingiber nimmonni from South India: Chemical characterization and antimicrobial activity. Phytochemistry 67: 2469-2473.

Sartoratto A, Machado ALM, Delarmelina C, Figueira GM, Duarte MCT, Redher VLG 2004. Composition and antimicrobial activity of essential oils from aromatic plants used in Brazil. Braz J Microbiol 35: 275-280.

Schelz ZMJ, Hohmann J 2006. Antimicrobial and antiplasmid activities of essential oils. Fitoterapia 77: 279-285.

Silva, MTN, Ushimaru PI, Barbosa LN, Cunha MLRS, Fernandes Junior A 2009 Antibacterial activity of plant essential oils against Staphylococcus aureus and Escherichia coli strains isolated from human specimens. Braz JMedicinal Plants 11: 257-262.

Silver LL, Bostian KA 1993. Discovery and development of new antibiotics: the problem of antibiotic resistance. Antimicrob Agents Ch 37: 377-383. 